\title{
Longitudinal exchange: an alternative strategy towards quantification of dynamics parameters in $\mathbf{Z Z}$ exchange spectroscopy
}

\author{
Karin Kloiber • Romana Spitzer • Sarina Grutsch • \\ Christoph Kreutz $\cdot$ Martin Tollinger
}

Received: 8 May 2011/ Accepted: 22 June 2011

(C) The Author(s) 2011. This article is published with open access at Springerlink.com

\begin{abstract}
Longitudinal exchange experiments facilitate the quantification of the rates of interconversion between the exchanging species, along with their longitudinal relaxation rates, by analyzing the time-dependence of direct correlation and exchange cross peaks. Here we present a simple and robust alternative to this strategy, which is based on the combination of two complementary experiments, one with and one without resolving exchange cross peaks. We show that by combining the two data sets systematic errors that are caused by differential linebroadening of the exchanging species are avoided and reliable quantification of kinetic and relaxation parameters in the presence of additional conformational exchange on the ms- $\mu$ s time scale is possible. The strategy is applied to a bistable DNA oligomer that displays different linebroadening in the two exchanging species.
\end{abstract}

Keywords Longitudinal exchange - DNA - Dynamics · Line-broadening $\cdot$ Relaxation

\section{Introduction}

Exchange experiments involving the transfer of heteronuclear longitudinal magnetization of product operator terms such as $I_{\mathrm{z}} S_{\mathrm{z}}$ or $S_{\mathrm{z}}$ were introduced by Montelione et al. to quantitate transitions between different conformations of biomolecules for which isolated resonances are observed (Montelione and Wagner 1989). These experiments have

K. Kloiber · R. Spitzer · S. Grutsch · C. Kreutz •

M. Tollinger $(\square)$

Institute of Organic Chemistry, University of Innsbruck,

Innrain 52a, 6020 Innsbruck, Austria

e-mail: martin.tollinger@uibk.ac.at played an important role in NMR dynamic studies of biological systems ever since, because they provide access to functionally important dynamic processes occurring on time scales ranging from $\sim 0.5$ to $\sim 50 \mathrm{~s}^{-1}$. In addition, they offer the possibility to resolve these processes on a per-residue basis and without perturbing the system from thermodynamic equilibrium, a feature that makes NMR spectroscopy an indispensable instrument for the analysis of macromolecular dynamics (Mittermaier and Kay 2009).

Longitudinal exchange experiments are based on the quantification of magnetization transfer between exchanging conformers during a mixing time. In the standard (frequency-labeled) setup, four peaks are observed for each spin, i.e. two direct correlation (diagonal) peaks and two exchange cross peaks (Fig. 1). In order to measure the interconversion rates between the exchanging conformers, a series of spectra is recorded for a range of mixing times, and the magnitudes of the four peaks are quantified by fitting the appropriate theoretical curves to the experimental data (Fig. 2) (Farrow et al. 1995). Several variants of this setup have been described, including 2D, 3D and pseudo-4D TROSY selected/detected versions (Hwang and Kay 2005; Sahu et al. 2007; Li and Palmer 2009; Sprangers et al. 2005; Nikolaev and Pervushin 2007; Wang et al. 2002) as well as zero-quantum/double quantum coherence versions to resolve chemical shift degeneracy (Robson et al. 2010).

The apparent simplicity of the analysis of longitudinal exchange experiments is, however, deceiving, as there are several pitfalls that have to be accounted for in order to avoid systematic errors. First, different relaxation properties of the two species result in different transfer efficiencies during INEPT step(s) (Tollinger et al. 2001; Miloushev et al. 2008). Second, and more importantly, the analysis of kinetic properties from this experiment may 

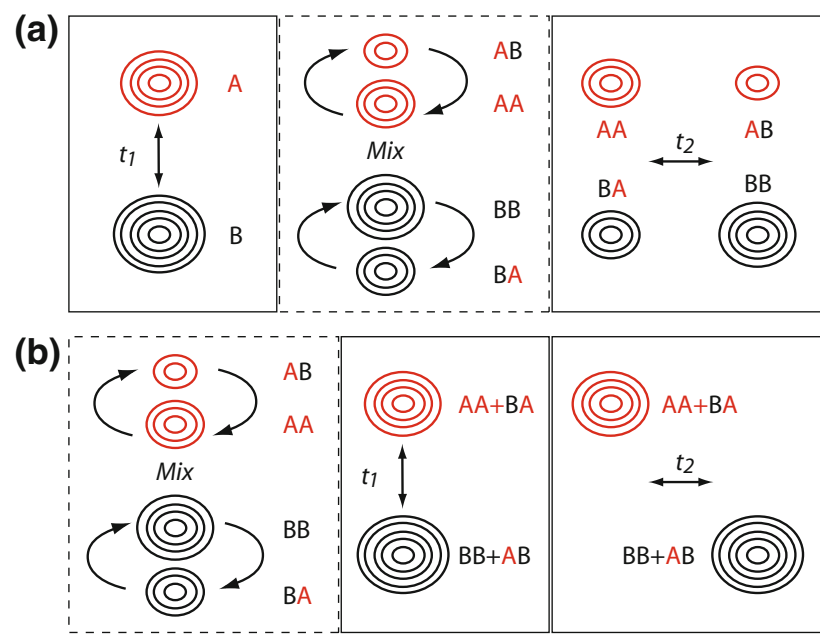

Fig. 1 a Scheme of a 2D longitudinal exchange experiment (Montelione and Wagner 1989). After frequency-labeling in an indirect dimension, $t_{1}$, magnetization associated with state A (B) (shown in red and black, respectively) either remains A (B) or is converted to B (A) during a variable mixing time (Mix). Subsequent frequencylabeling in the direct dimension $\left(t_{2}\right)$ gives rise to two direct correlation (AA and $\mathrm{BB}$ ) and two exchange cross peaks (AB, BA), respectively. b If the order of chemical shift evolution $\left(t_{1}\right)$ and mixing times is interchanged, exchange cross peaks are not resolved but remain part of the two direct correlation peaks $\mathrm{A}$ and $\mathrm{B}$, which corresponds to the collapse of $\mathrm{AA}$ with $\mathrm{BA}$ and $\mathrm{BB}$ with $\mathrm{AB}$, respectively

suffer from substantial systematic errors if the line-widths of the two species are different (Fig. 3). Unless precautions are taken to ensure precise determination of peak volumes, errors due to systematic over- or underestimation of the magnitudes of exchange cross peaks can have a significant impact on the fitting results.

Because determining peak volumes by integration requires fitting to a functional form, this is particularly challenging in cases where non-canonical line-shapes (resulting from conformational exchange processes occurring on the ms- $\mu$ s time scale) are encountered, which are not known a priori. Moreover, it can sometimes be impractical to precisely measure the volumes of small exchange cross peaks with low signal-to-noise ratio, as typically encountered for short mixing times, in particular in the vicinity of strong direct correlation peaks. To circumvent integration, peak volumes are often approximated by partial peak volumes that are obtained by summation of the intensities of data points within a defined interval. Partial peak volumes are, however, dependent on the choice of the summation interval with respect to the linewidth (Rischel 1995), thereby complicating the normalization of peaks with different line-widths. To improve the precision in the determination of longitudinal exchange kinetics, a quadratic approximation was proposed for the analysis of the data by employing a composite ratio of exchange and direct correlation peak intensities. This approach does, however, require that equilibrium constants can be determined from other experiments (Miloushev et al. 2008).

Here we present a simple and robust alternative to the standard setup of multidimensional longitudinal exchange experiments for biomolecules. Our approach is based on collecting two complementary data sets, one where exchange cross peaks are resolved, and a second one where they are not. This strategy is reminiscent of (one-dimensional) approaches comparing selective and non-selective inversion recovery measurements, as pioneered by Hoffman and Forsen (1966a, b). Combining the two data sets eliminates systematic errors that can be caused by differential line-broadening and enables reliable quantification of kinetic and relaxation parameters. As an example, we have used this experimental setup to characterize conformational exchange in a selectively ${ }^{13} \mathrm{C}$ labeled sample of a bistable DNA oligomer.

\section{Materials and methods}

Sample preparation and conditions

A sample of bistable DNA (TCGTACCGGAAGGTAC GAACCTTCCG) (Puffer et al. 2009) was synthesized and purified as described (Kloiber et al. 2011), introducing isolated methyl $\left({ }^{13} \mathrm{CHD}_{2}\right)$ labels into thymidines to avoid complications due to cross-correlated relaxation in the methyl group. The NMR sample contained 1.0 mM DNA, $50 \mathrm{mM}$ sodium arsenate buffer, $\mathrm{pH} 6.5$, and $100 \% \mathrm{D}_{2} \mathrm{O}$, All NMR spectra were recorded at $33^{\circ} \mathrm{C}$.

\section{NMR measurements}

2-Dimensional longitudinal exchange experiments were recorded using pulse sequences that are based on the scheme described by Farrow et al., but employing the exchange of two-spin heteronuclear order, $I_{Z} S_{Z}$ (Farrow et al. 1995). In one experiment ${ }^{13} \mathrm{C}$ frequency labeling preceded the mixing time, while in the second experiment the order of indirect evolution $\left({ }^{13} \mathrm{C}\right.$ frequency labeling $)$ and mixing times was interchanged. Deuterium decoupling was employed during ${ }^{13} \mathrm{C}\left(t_{1}\right)$ and ${ }^{1} \mathrm{H}\left(t_{2}\right)$ detection. NMR spectra were recorded on a Varian Inova spectrometer operating at a Larmor frequency of $500 \mathrm{MHz}$. Series of 10 2D spectra (with and without frequency labeling) were collected, with mixing times ranging from 10 to $800 \mathrm{~ms}$. Spectra were recorded as complex data matrices composed of $512 \times 38$ points, with maximum acquisition times of 127 and $64 \mathrm{~ms}$ in the ${ }^{13} \mathrm{C}$ and ${ }^{1} \mathrm{H}$ dimensions, respectively. 32 scans per FID were obtained with a recycle delay of $2 \mathrm{~s}$, resulting in a total experimental time of $18.5 \mathrm{~h}$ for each series. 

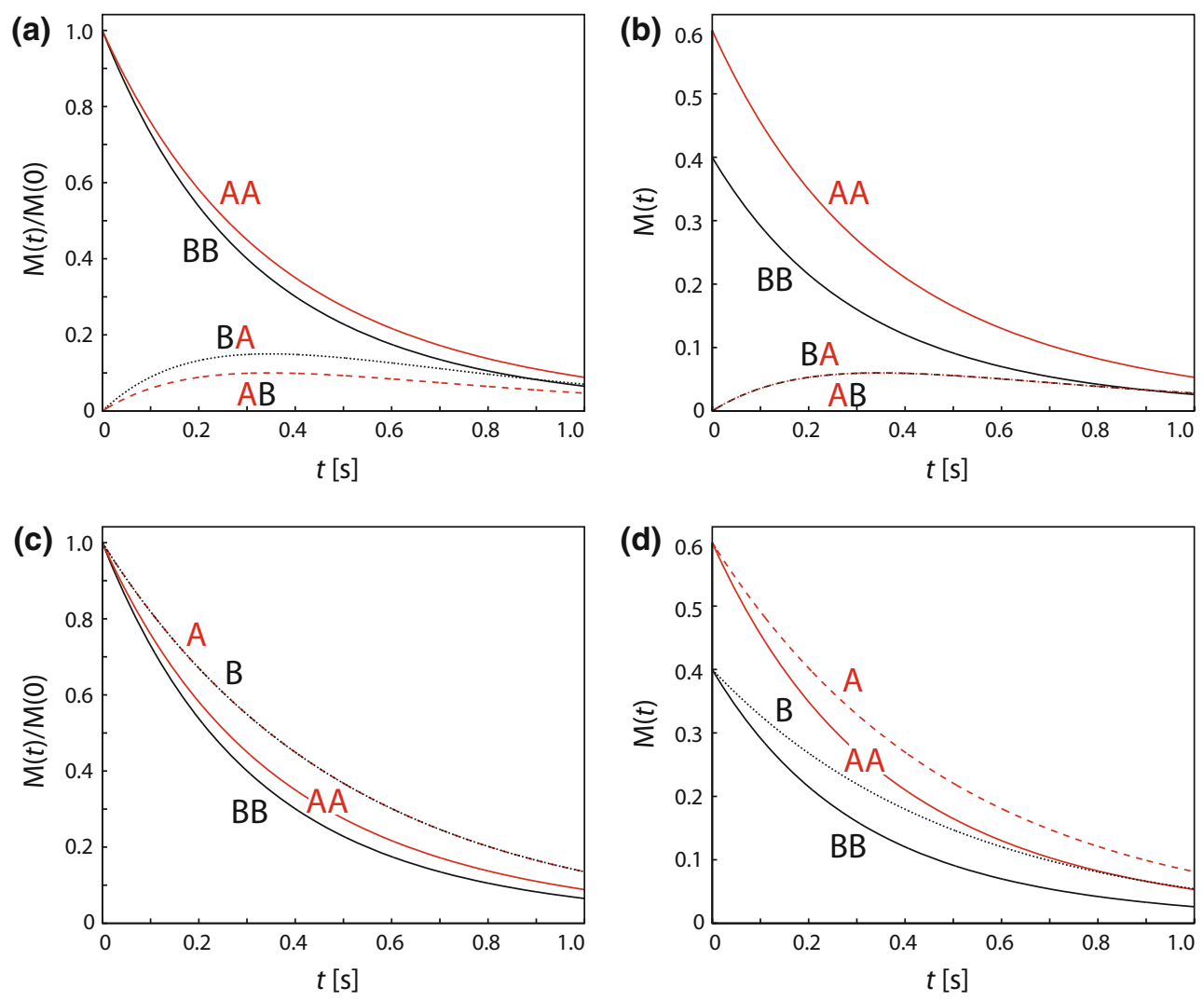

Fig. 2 a Time dependence of normalized direct correlation peaks AA and $\mathrm{BB}$ (red and black solid lines, respectively) and exchange cross peaks $\mathrm{AB}$ and $\mathrm{BA}$ (red and black dashed lines), simulated using (1) and (2). Parameters: $k_{\mathrm{AB}}=0.8 \mathrm{~s}^{-1}, k_{\mathrm{BA}}=1.2 \mathrm{~s}^{-1}, R_{1}^{\mathrm{A}}=2.0 \mathrm{~s}^{-1}$, $R_{1}^{\mathrm{B}}=2.0 \mathrm{~s}^{-1}$. b As in a, but without normalization by $\mathrm{M}_{\mathrm{AA}}(0)$ and $\mathrm{M}_{\mathrm{BB}}(0)$ (but $\mathrm{M}_{\mathrm{AA}}(0)+\mathrm{M}_{\mathrm{BB}}(0)$ set to 1$)$. Exchange cross peaks have equal magnitudes if $R_{1}^{\mathrm{A}}=R_{1}^{\mathrm{B}}$. c Time dependence of normalized direct correlation peaks A and B [(3), red and black dashed lines], as

well as AA and BB [(1), red and black solid lines], using the same parameters as in a. The curves for $\mathrm{A}$ and $\mathrm{B}$ are identical if $R_{1}^{\mathrm{A}}=R_{1}^{\mathrm{B}}$. By taking the difference between $\mathrm{A}$ and $\mathrm{AA}$ (B and $\mathrm{BB}$ ) curves it is possible to reconstruct the correctly normalized exchange curves $\mathrm{AB}$ (BA) shown in a. $\mathbf{d}$ As in $\mathbf{c}$ but without normalization by $\mathrm{M}_{\mathrm{A}}(0)$, $\mathrm{M}_{\mathrm{AA}}(0)$ and $\mathrm{M}_{\mathrm{B}}(0), \mathrm{M}_{\mathrm{BB}}(0)$, respectively. Here, the differences between $\mathrm{A}$ and $\mathrm{AA}(\mathrm{B}$ and $\mathrm{BB})$ are equal

\section{Data analysis}

Data were processed and analyzed using NMRPipe and nmrDraw software (Delaglio et al. 1995) and in-house written fitting scripts. The $t_{1}\left(t_{2}\right)$ time domain data were apodized using a Gaussian function, linear predicted to 128 data points $\left(t_{1}\right)$, and zero filled to $4,096 \times 256$ points. Partial peak volumes were obtained by adding the intensities in boxes of $7 \times 7,5 \times 5$ or $3 \times 3$ data points centered on the peak maximum. A least-squares fitting procedure was employed to extract $k_{\mathrm{AB}}, k_{\mathrm{BA}}$ and $R_{1}^{\mathrm{A}}, R_{1}^{\mathrm{B}}$ by fitting the expressions given in (1) and (3) to the measured partial peak volumes, along with $\mathrm{M}_{\mathrm{AA}}(0), \mathrm{M}_{\mathrm{BB}}(0)$ and $\mathrm{M}_{\mathrm{A}}(0), \mathrm{M}_{\mathrm{B}}(0)$ values, respectively. For comparison, the two direct correlation peaks along with the single resolved exchange cross peak for each spin label were used to extract $k_{\mathrm{AB}}, k_{\mathrm{BA}}$ and $R_{1}^{\mathrm{A}}, R_{1}^{\mathrm{B}}$ by fitting the appropriate expressions given in (1) and (2) to the measured partial

peak volumes $(3 \times 3$ data points). In all cases, experimental uncertainties were estimated via a Monte Carlo approach in which 200 synthetic data sets were generated from the best-fit values of $k_{\mathrm{AB}}, k_{\mathrm{BA}}$ and $R_{1}^{\mathrm{A}}, R_{1}^{\mathrm{B}}$ by adding random errors based on the signal-to-noise ratio to the bestfit curves. Errors quoted in the paper are the standard deviations in fitted parameters that were obtained in this procedure.

\section{Results and discussion}

Our approach relies on recording two separate experiments. The first one is analogous to the two-dimensional experiment described by Montelione et al., where heteronuclear chemical shift evolution precedes the mixing time and four resonances, two direct correlation and two exchange cross peaks, all frequency-labeled in both 
dimensions, are observed for each spin (Montelione and Wagner 1989). In the second experiment, the order of chemical shift evolution and mixing times is interchanged, so that exchange cross peaks are not resolved but remain part of the corresponding direct correlation peaks (Fig. 1). Analysis of the time dependence of only the direct correlation peaks in the two experiments with and without frequency-labeling eliminates systematic errors that arise from differential line broadening effects, as described below.

In the frequency-labeled experiment, the time dependence of the direct correlation peaks of a two-site exchanging system is given by (Farrow et al. 1995; Palmer et al. 2001)

$$
\begin{aligned}
\mathrm{M}_{\mathrm{BB}}(t) / \mathrm{M}_{\mathrm{BB}}(0)= & \frac{1}{\left(\lambda_{1}-\lambda_{2}\right)} \\
& {\left[\left(a_{11}+\lambda_{1}\right) \cdot e^{\lambda_{1} t}-\left(a_{11}+\lambda_{2}\right) \cdot e^{\lambda_{2} t}\right] } \\
\mathrm{M}_{\mathrm{AA}}(t) / \mathrm{M}_{\mathrm{AA}}(0)= & \frac{1}{\left(\lambda_{1}-\lambda_{2}\right)} \\
& {\left[\left(a_{22}+\lambda_{1}\right) \cdot e^{\lambda_{1} t}-\left(a_{22}+\lambda_{2}\right) \cdot e^{\lambda_{2} t}\right] }
\end{aligned}
$$

where $\mathrm{M}_{\mathrm{AA}}$ and $\mathrm{M}_{\mathrm{BB}}$ are the magnitudes of the direct correlation peaks corresponding to sites $\mathrm{A}$ and $\mathrm{B}$, which are normalized by the amount of magnetization associated with states $\mathrm{A}$ and $\mathrm{B}$ (the magnitudes of the direct correlation peaks at the start of the mixing period, $\mathrm{M}_{\mathrm{AA}}(0)$ and $\mathrm{M}_{\mathrm{BB}}(0)$, respectively). The eigenvalues of the spin density matrix which includes magnetization transfer effects due to chemical exchange, $\lambda_{1 / 2}$, are given by $\lambda_{1 / 2}=1 / 2$ $\left[-\left(a_{11}+\mathrm{a}_{22}\right) \pm\left[\left(a_{11}-a_{22}\right)^{2}+4 a_{12} a_{21}\right]^{1 / 2}\right]$, where elements $a_{\mathrm{ij}}$ are defined as $a_{11}=k_{\mathrm{AB}}+R_{1}^{\mathrm{A}}, a_{22}=$ $k_{\mathrm{BA}}+R_{1}^{\mathrm{B}}, a_{12}=-k_{\mathrm{BA}}$ and $a_{21}=-k_{\mathrm{AB}}$. Parameters $k_{\mathrm{AB}}$ and $k_{\mathrm{BA}}$ denote the microscopic kinetic rate constants that describe the interconversion between states $\mathrm{A}$ and $\mathrm{B}$, while $R_{1}^{\mathrm{A}}$ and $R_{1}^{\mathrm{B}}$ are the longitudinal relaxation rates of magnetization in sites $\mathrm{A}$ and $\mathrm{B}$. Likewise, the normalized magnitudes of the associated exchange cross peaks in the frequency-labeled experiment are given by (Farrow et al. 1995; Palmer et al. 2001)

$$
\begin{aligned}
& \mathrm{M}_{\mathrm{AB}}(t) / \mathrm{M}_{\mathrm{AA}}(0)=\frac{1}{\left(\lambda_{1}-\lambda_{2}\right)}\left[-a_{21} \cdot e^{\lambda_{1} t}+a_{21} \cdot e^{\lambda_{2} t}\right] \\
& \mathrm{M}_{\mathrm{BA}}(t) / \mathrm{M}_{\mathrm{BB}}(0)=\frac{1}{\left(\lambda_{1}-\lambda_{2}\right)}\left[-a_{12} \cdot e^{\lambda_{1} t}+a_{12} \cdot e^{\lambda_{2} t}\right] .
\end{aligned}
$$

In the experiment without frequency labeling only two direct correlation peaks are observed, one of them with a magnitude, $\mathrm{M}_{\mathrm{A}}$, that corresponds to the sum of $\mathrm{AA}$ and $A B$, while the magnitude of the second peak, $M_{B}$, results from $\mathrm{BB}$ and $\mathrm{AB}$. The normalized dependencies of $\mathrm{M}_{\mathrm{A}}$ and $\mathrm{M}_{\mathrm{B}}$ are then given by

$$
\begin{aligned}
\mathrm{M}_{\mathrm{A}}(t) / \mathrm{M}_{\mathrm{A}}(0) & =\frac{1}{\left(\lambda_{1}-\lambda_{2}\right)} \\
& {\left[\left(a_{22}-a_{21}+\lambda_{1}\right) \cdot e^{\lambda_{1} t}-\left(a_{22}-a_{21}+\lambda_{2}\right) \cdot e^{\lambda_{2} t}\right] } \\
\mathrm{M}_{\mathrm{B}}(t) / \mathrm{M}_{\mathrm{B}}(0) & =\frac{1}{\left(\lambda_{1}-\lambda_{2}\right)} \\
& {\left[\left(a_{11}-a_{12}+\lambda_{1}\right) \cdot e^{\lambda_{1} t}-\left(a_{11}-a_{12}+\lambda_{2}\right) \cdot e^{\lambda_{2} t}\right] }
\end{aligned}
$$

where $\mathrm{M}_{\mathrm{A}}(0)$ and $\mathrm{M}_{\mathrm{B}}(0)$ are the magnitudes of the peaks $\mathrm{A}$ and $\mathrm{B}$ at zero mixing time.

Using the standard frequency-labeled strategy to extract $R_{1}^{\mathrm{A}}, R_{1}^{\mathrm{B}}$ and $k_{\mathrm{AB}}, k_{\mathrm{BA}}$ involves simultaneous least-squares fitting of (1) and (2) to exchange cross peaks and direct correlation peaks (Fig. 2). It is obvious from (2), however, that this requires the normalization of the two exchange cross peaks $\mathrm{M}_{\mathrm{AB}}(\mathrm{t}), \mathrm{M}_{\mathrm{BA}}(\mathrm{t})$ by the direct correlation peaks $\mathrm{M}_{\mathrm{AA}}(0), \mathrm{M}_{\mathrm{BB}}(0)$, respectively. Because $\mathrm{M}_{\mathrm{AB}}$ and $\mathrm{M}_{\mathrm{AA}}$ (as well as $\mathrm{M}_{\mathrm{BA}}$ and $\mathrm{M}_{\mathrm{BB}}$ ) have different line widths in the direct $\left(t_{2}\right)$ dimension in most cases (see Fig. 3), special care has to be taken to ensure precise determination of peak volumes in order to avoid errors due to systematic over- or underestimation of exchange cross peak magnitudes, and concomitant over- or underestimation of the corresponding kinetic rate constants.

This complication can be avoided by using the four direct correlation peaks in experiments with and without frequency-labeling, $\mathrm{M}_{\mathrm{AA}}, \mathrm{M}_{\mathrm{BB}}$ and $\mathrm{M}_{\mathrm{A}}, \mathrm{M}_{\mathrm{B}}$, respectively, for the analysis of longitudinal exchange. In this case all peaks are normalized by their respective magnitudes at the start of the mixing period, $\mathrm{M}_{\mathrm{AA}}(0), \mathrm{M}_{\mathrm{BB}}(0)$ and $\mathrm{M}_{\mathrm{A}}(0)$, $\mathrm{M}_{\mathrm{B}}(0)$, with inherently identical line widths. Thus, longitudinal relaxation and kinetic rate constants $\left(R_{1}^{\mathrm{A}}, R_{1}^{\mathrm{B}}\right.$ and $\left.k_{\mathrm{AB}}, k_{\mathrm{BA}}\right)$ can be extracted from the four direct correlation peaks by simultaneous fitting of (1) and (3) to the experimental data, yielding values that are not distorted by differential line-broadening effects. Consequently, the results are independent of the peak integration method, i.e. intensities, partial peak volumes or (exact) peak volumes can be used.

We have applied this simple strategy to a bistable DNA 26-mer that exists in two different conformational (hairpin) sub-states A and B, which interconvert slowly on the NMR chemical shift time scale (Puffer et al. 2009). Both states are populated to a considerable extent at ambient temperature and in aqueous buffer $(\mathrm{pH}$ 6.5), and discrete sets of resonances are observed for $\mathrm{A}$ and $\mathrm{B}$ (Fig. 4). Selective ${ }^{13} \mathrm{C}$ labeling $\left({ }^{13} \mathrm{CHD}_{2}\right.$-methyl) in thymidines enables the measurement of longitudinal exchange (2-spin order) between folds $\mathrm{A}$ and $\mathrm{B}$ in time series of 2-dimensional experiments performed with and without ${ }^{13} \mathrm{C}$ frequency-labeling, respectively. Two thymidines, $\mathrm{T} 4$ and $\mathrm{T} 22$, were chosen as probes for the interconversion process between the two sub-states because the T22 spin label clearly displays 


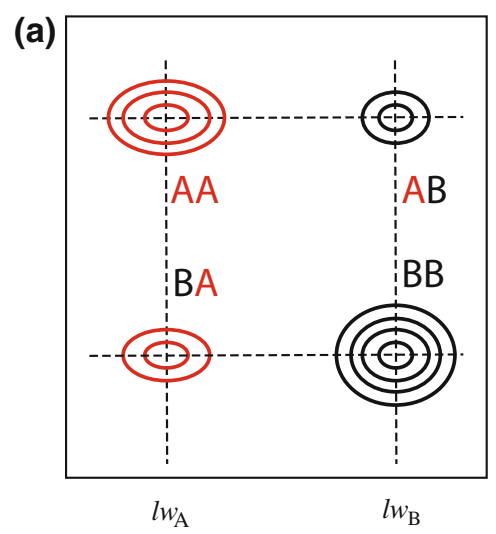

Fig. 3 Effect of differential line-broadening in 2D frequency-labeled experiments. a Peaks associated with state A during $t_{2}$ detection (AA direct correlation and BA exchange cross peaks) have identical line widths $\left(l w_{\mathrm{A}}\right)$ in the direct dimension, which can be different from that of $\mathrm{BB}$ and $\mathrm{AB}\left(l w_{\mathrm{B}}\right)$. Because quantification of $\mathrm{AB}(\mathrm{BA})$ exchange cross peaks requires normalization by $\mathrm{AA}(\mathrm{BB})$ direct correlation peaks at zero mixing time, exact peak volumes rather than intensities or partial peak volumes have to be used for analysis of the data. Differences in line-widths during $t_{1}$ evolution are irrelevant for the analysis. b Simulation of the effect of approximating peak volumes by

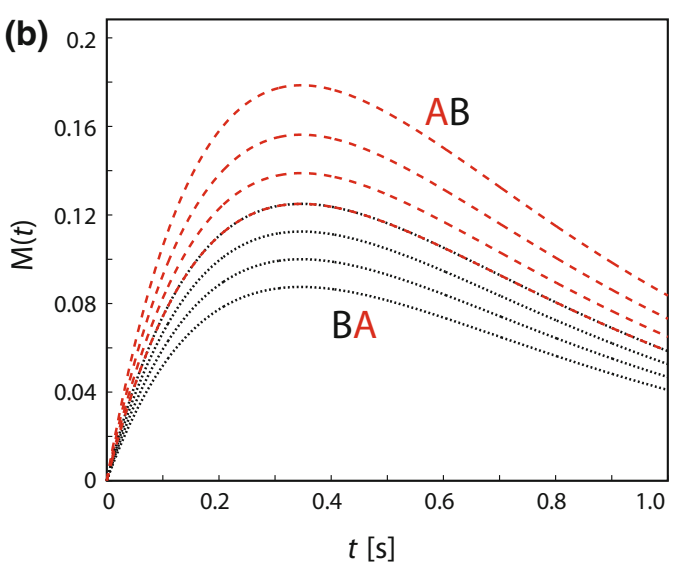

intensities for normalized exchange curves, using (2). Parameters: $k_{\mathrm{AB}}=1.0 \mathrm{~s}^{-1}, k_{\mathrm{BA}}=1.0 \mathrm{~s}^{-1}, R_{1}^{\mathrm{A}}=2.0 \mathrm{~s}^{-1}, R_{1}^{\mathrm{B}}=2.0 \mathrm{~s}^{-1}$. Assuming Lorentzian line shapes (where $\mathrm{Int}=\mathrm{Vol} / l w$ ), normalized exchange curves based on intensity measurements are simulated for $l w_{\mathrm{A}} / l w_{\mathrm{B}}=0.7,0.8,0.9$, and 1.0. $\mathrm{AB}$ and $\mathrm{BA}$ exchange curves are shown in red and black, respectively. Only if $l w_{\mathrm{A}}=l w_{\mathrm{B}}$, or if exact (direct correlation and exchange cross peak) volumes are used, $\mathrm{AB}$ and $\mathrm{BA}$ exchange curves are identical, as is expected for $k_{\mathrm{AB}}=k_{\mathrm{BA}}$ (superimposed red and black dashed lines) (a)
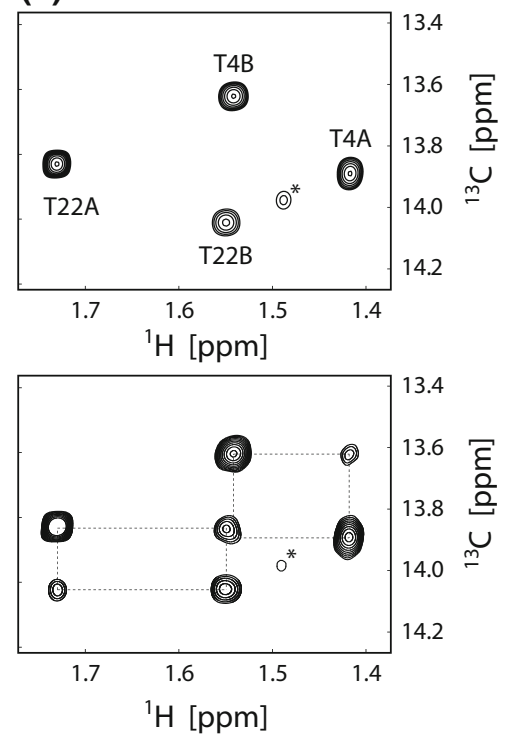

(b)
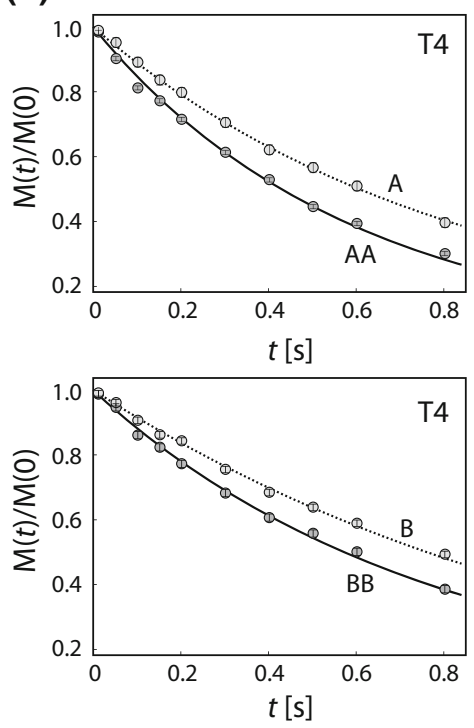
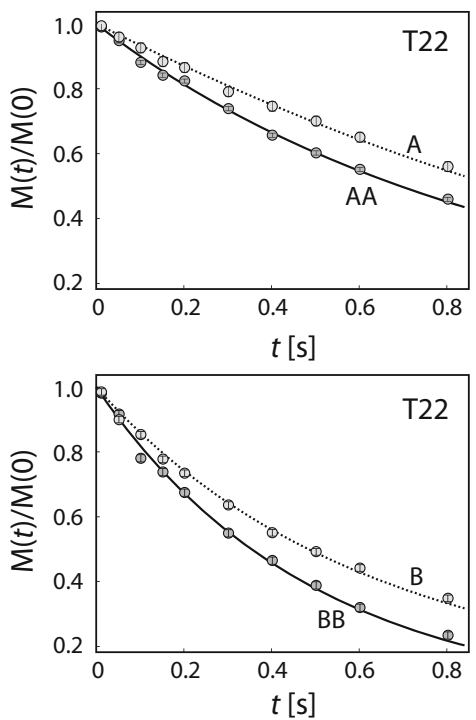

Fig. 4 Experimental longitudinal exchange data recorded for (T4, T22)-methyl ${ }^{13} \mathrm{C}$ labeled bistable DNA. a Thymidine methyl group region of a 2-dimensional ${ }^{1} \mathrm{H}^{13} \mathrm{C}$ correlation spectrum without (top) and with (bottom) frequency-labeling in $\left(t_{1}\right)$ (mixing time $120 \mathrm{~ms}$ ). Direct correlation peaks of T4 and T22 are labeled. Duplex DNA, which does not participate in the exchange process, is indicated by an asterisk. b Normalized partial peak volumes of A, B, AA and BB direct correlation peaks of spin labels $\mathrm{T} 4$ and $\mathrm{T} 22$, together with bestfit curves (dashed lines for A, B, solid lines for AA, BB). All four

differential line-broadening in folds $\mathrm{A}$ and $\mathrm{B}$, consistent with the presence of an A-T/A-T step (A10-T23/A11-T24) in fold B (Lingbeck et al. 1996). Of note, the AB exchange curves ((1) and (3)) were fitted simultaneously to the experimental data for each spin label to yield kinetic rate constants $k_{\mathrm{AB}}=$ $0.51 \pm 0.08 \mathrm{~s}^{-1}$ (T4), $k_{\mathrm{AB}}=0.42 \pm 0.07 \mathrm{~s}^{-1}$ (T22), $k_{\mathrm{BA}}=0.41 \pm$ $0.07 \mathrm{~s}^{-1}(\mathrm{~T} 4), k_{\mathrm{BA}}=0.49 \pm 0.06 \mathrm{~s}^{-1}$ (T22), and longitudinal relaxation rate constants $R_{1}^{\mathrm{A}}=1.21 \pm 0.04 \mathrm{~s}^{-1}(\mathrm{~T} 4), R_{1}^{\mathrm{A}}=0.67 \pm$ $0.05 \mathrm{~s}^{-1}$ (T22), $R_{1}^{\mathrm{B}}=0.90 \pm 0.04 \mathrm{~s}^{-1} \quad$ (T4) and $R_{1}^{\mathrm{B}}=1.59 \pm$ $0.04 \mathrm{~s}^{-1}$ (T22). Uncertainties were derived from a MonteCarlo approach

cross peaks of T4 and T22 overlap at $500 \mathrm{MHz}$ and $33^{\circ} \mathrm{C}$ in the ${ }^{13} \mathrm{CHD}_{2}$-methyl labeled sample, preventing straightforward analysis of the interconversion process between 
folds $\mathrm{A}$ and $\mathrm{B}$ by the standard (frequency-labeled) approach.

Figure 4 shows the experimental data obtained for the AA, BB, A and B direct correlation peaks in experiments with and without frequency-labeling, together with best-fit curves using (1) and (3). Kinetic rate constants of $k_{\mathrm{AB}}=0.51 \pm 0.08 \mathrm{~s}^{-1}$ (T4) and $k_{\mathrm{AB}}=0.42 \pm 0.07 \mathrm{~s}^{-1}$ (T22), as well as $k_{\mathrm{BA}}=0.41 \pm 0.07 \mathrm{~s}^{-1}$ (T4) and $k_{\mathrm{BA}}=0.49 \pm 0.06 \mathrm{~s}^{-1}$ (T22) were obtained, with uncertainties derived from a MonteCarlo approach. Despite the considerable difference in line-broadening between folds A and B for T22, the extracted kinetic rates for this spin label are in good agreement with those of $\mathrm{T} 4$, which is consistent with a collective two-state conformational transition between the two folds. As expected, the extracted parameters do not depend on the peak integration method: using partial peak volumes by adding the intensities in $7 \times 7$ grids centered on the peak maximum yields values that are identical (within experimental uncertainties) to the ones obtained using $5 \times 5$ or $3 \times 3$ grids, or by using peak intensities.

For comparison, the frequency-labeled data set was used to determine kinetic rate constants by analyzing the single (BA) exchange cross peak that is resolved for each spin label along with its two direct correlation peaks. In this case, only values for one rate constant $\left(k_{\mathrm{BA}}\right)$ could be determined reliably. Only for the T4 spin label the value of $k_{\mathrm{BA}}\left(0.58 \pm 0.10 \mathrm{~s}^{-1}\right)$ is in good agreement with the value obtained by analyzing $\mathrm{AA}, \mathrm{BB}, \mathrm{A}$ and $\mathrm{B}$ direct correlation peaks, while for T22 the magnitudes of normalized BA exchange cross peaks are overestimated due to considerable line-broadening of fold $\mathrm{B}$, which results in an increase of $k_{\mathrm{BA}}\left(1.17 \pm 0.21 \mathrm{~s}^{-1}\right)$.

It is evident from (1) and (3) and Fig. 2 that the biexponential decays of $\mathrm{A}$ and $\mathrm{B}$ direct correlation peaks differ from those of the AA and BB direct correlation peaks by an amount that corresponds to the pertinent exchange peak. ${ }^{1}$ Thus, subtraction of normalized AA intensities from A (and $\mathrm{BB}$ intensities from B) can, in principle, be employed to formally reconstruct the (correctly!) normalized magnitudes of the corresponding exchange cross peaks $\mathrm{AB}$ (BA) that are not affected by differential line-broadening, as they are computed from two peaks with identical line-widths, and irrespective of cross peak overlap. These exchange cross peak intensities can then be analyzed, together with AA and BB direct correlation peaks, as in the conventional frequency-labeled approach using (1) and (2) to obtain longitudinal exchange and kinetic rate constants. We opt not to do

\footnotetext{
1 This feature was employed by Wider et al. (1991) to facilitate the observation of small exchange cross peaks in difference correlation spectra with minimal interference from direct correlation peaks.
}

so, however, since analysis of the four direct correlation peaks directly is robust and straightforward.

\section{Summary}

We have presented an alternative way of analyzing longitudinal exchange in two-state exchanging systems by recording pairs of two- (or more) dimensional spectra with and without frequency-labeling in an indirect dimension. Analysis of the time-dependence of the four $(2+2)$ direct correlation peaks that are observed in the two experiments enables reliable and robust quantification of the interconversion rates between the two species, along with their longitudinal relaxation rates. This approach partly resolves the issue of cross peak overlap and eliminates errors caused by differential line-broadening of the two species, thereby expanding the applicability of longitudinal exchange experiments to systems with additional conformational exchange on the ms- $\mu$ s time scale.

While our approach increases the experimental time by a factor of two, it significantly reduces the number of sources for systematic errors. We recommend to generally use this strategy in cases where (1) resonances show different line broadening, or (2) exchange cross peaks are overlapped or close to intense direct correlation peaks (which may preclude the determination of their exact volumes).

Acknowledgments This work was supported by the Austrian Science Fund FWF (KK: V173, MT: P22735).

Open Access This article is distributed under the terms of the Creative Commons Attribution Noncommercial License which permits any noncommercial use, distribution, and reproduction in any medium, provided the original author(s) and source are credited.

\section{References}

Delaglio F, Grzesiek S, Vuister GW, Zhu G, Pfeifer J, Bax A (1995) NMRPipe: a multidimensional spectral processing system based on UNIX pipes. J Biomol NMR 6:277-293

Farrow NA, Zhang O, Forman-Kay JD, Kay LE (1995) Comparison of the backbone dynamics of a folded and an unfolded $\mathrm{SH} 3$ domain existing in equilibrium in aqueous buffer. Biochemistry 34(3):868-878

Hoffman RA, Forsen S (1966a) High resolution nuclear magnetic double and multiple resonance. Prog Nucl Magn Reson 1(1966): 15-204

Hoffman RA, Forsen S (1966b) Transient and steady-state Overhauser experiments in the investigation of relaxation processes. Analogies between chemical exchange and relaxation. J Chem Phys 45(6):2049-2060

Hwang PM, Kay LE (2005) Solution structure and dynamics of integral membrane proteins by NMR: a case study involving the enzyme PagP. Methods Enzymol 394:335-350 
Kloiber K, Spitzer R, Tollinger M, Konrat R, Kreutz C (2011) Probing RNA dynamics via longitudinal exchange and CPMG relaxation dispersion NMR spectroscopy using a sensitive ${ }^{13} \mathrm{C}$ methyl label. Nucleic Acids Res 39(10):4340-4351

Li Y, Palmer AG III (2009) TROSY-selected ZZ-exchange experiment for characterizing slow chemical exchange in large proteins. J Biomol NMR 45(4):357-360

Lingbeck J, Kubinec MG, Miller J, Reid BR, Drobny GP, Kennedy MA (1996) Effect of adenine methylation on the structure and dynamics of TpA steps in DNA: NMR structure determination of [d(CGAGGTTTAAACCTCG)]2 and its A9-methylated derivative at $750 \mathrm{MHz}$. Biochemistry 35(3):719-734

Miloushev VZ, Bahna F, Ciatto C, Ahlsen G, Honig B, Shapiro L, Palmer AG III (2008) Dynamic properties of a type II cadherin adhesive domain: implications for the mechanism of strandswapping of classical cadherins. Structure 16(8):1195-1205

Mittermaier AK, Kay LE (2009) Observing biological dynamics at atomic resolution using NMR. Trends Biochem Sci 34(12): $601-611$

Montelione GT, Wagner G (1989) 2D chemical exchange NMR spectroscopy by proton-detected heteronuclear correlation. J Am Chem Soc 111:3096-3098

Nikolaev Y, Pervushin K (2007) NMR spin state exchange spectroscopy reveals equilibrium of two distinct conformations of leucine zipper GCN4 in solution. J Am Chem Soc 129(20):6461-6469

Palmer AG III, Kroenke CD, Loria JP (2001) Nuclear magnetic resonance methods for quantifying microsecond-to-millisecond motions in biological macromolecules. Methods Enzymol 339: 204-238

Puffer B, Kreutz C, Rieder U, Ebert MO, Konrat R, Micura R (2009) 5-Fluoro pyrimidines: labels to probe DNA and RNA secondary structures by 1D 19F NMR spectroscopy. Nucleic Acids Res 37(22):7728-7740

Rischel C (1995) Fundamentals of peak integration. J Magn Reson A 116:255-258

Robson SA, Peterson R, Bouchard LS, Villareal VA, Clubb RT (2010) A heteronuclear zero quantum coherence Nz-exchange experiment that resolves resonance overlap and its application to measure the rates of heme binding to the IsdC protein. J Am Chem Soc 132(28):9522-9523

Sahu D, Clore GM, Iwahara J (2007) TROSY-based z-exchange spectroscopy: application to the determination of the activation energy for intermolecular protein translocation between specific sites on different DNA molecules. J Am Chem Soc 129(43): 13232-13237

Sprangers R, Gribun A, Hwang PM, Houry WA, Kay LE (2005) Quantitative NMR spectroscopy of supramolecular complexes: dynamic side pores in ClpP are important for product release. Proc Natl Acad Sci USA 102(46):16678-16683

Tollinger M, Skrynnikov NR, Mulder FA, Forman-Kay JD, Kay LE (2001) Slow dynamics in folded and unfolded states of an SH3 domain. J Am Chem Soc 123(46):11341-11352

Wang H, He Y, Kroenke CD, Kodukula S, Storch J, Palmer AG, Stark RE (2002) Titration and exchange studies of liver fatty acidbinding protein with $13 \mathrm{C}$-labeled long-chain fatty acids. Biochemistry 41(17):5453-5461

Wider G, Neri D, Wüthrich K (1991) Studies of slow conformational equilibria in macromolecules by exchange of heteronuclear longitudinal 2-spin-order in a 2D difference correlation experiment. J Biomol NMR 1:93-98 